\title{
Master international double diplômant entre la France et la Chine sur la base du processus de Bologne
}

\author{
Olivier BONNAUD, Lotfi SENHADJI \\ Professeurs à l’Université de Rennes 1 \\ Responsables respectifs du parcourt International du Master Électronique et Télécommunications, \\ Spécialités « Microélectronique et Signal \& Images », UFR ISTIC, Université de Rennes 1, Rennes, France. \\ Huazhong SHU, Lei WEI \\ Professeurs à l'Université du Sud-Est (Nankin) \\ Responsables respectifs du parcourt International du Master Électronique et Télécommunications \\ Spécialités « Microélectronique et Signal \& Images », SouthEast University, Nanjing, Chine.
}

\begin{abstract}
RESUME : En raison de la diminution du nombre d'étudiants en France dans le domaine de l'EEA, ce qui est de plus en plus fréquent en Europe, plusieurs établissements d'enseignement supérieur français ont bâti des formations délocalisées, notamment dans la zone Asie-Pacifique. Cet article traite de la mise en place d'un master dans le domaine de l’Électronique et Télécommunications, avec deux spécialités, la première en microélectronique et la seconde en traitement du signal et de l'image. C'est une formation aboutissant à un double-diplôme entre une université chinoise, la South-East University (SEU) à Nankin (Chine) et l’Université de Rennes 1, à Rennes (France). Ce master est entièrement compatible avec les règles du processus de Bologne plus particulièrement en termes de crédits (ECTS). Cette formation fonctionne depuis plus de deux ans et les premières promotions sont sorties. Cet article donne des informations sur la richesse de cette approche et l'intérêt pédagogique à la fois pour les étudiants mais aussi pour les enseignants des deux institutions partenaires.
\end{abstract}

Mots clés : master international, processus de Bologne, retour d'expérience.

\section{INTRODUCTION.}

En raison de la diminution du nombre d'étudiants en France dans le domaine de l'EEA (électronique, électrotechnique, automatique, signal, image) ce qui est de plus en plus fréquent en Europe, plusieurs établissements d'enseignement supérieur français ont bâti des formations délocalisées, notamment dans la zone AsiePacifique.

Dans cette nouvelle approche, des étudiants sont inscrits dans ces domaines, suivent des enseignements de qualité équivalente et peuvent en fin de master alimenter les écoles doctorales grâce à la préparation de thèses qui peuvent être en co-tutelle et de la sorte accroître le potentiel humain de recherche de nos laboratoires respectifs.

Ces formations donnent aussi l'opportunité d'établir ou d'amplifier les échanges scientifiques entre les deux établissements principalement au niveau des laboratoires de recherche concernés.

Cet article traite de la mise en place d'un master dans le domaine de l'Électronique et des Télécommunications, avec deux spécialités, la première en microélectronique et la seconde en traitement du signal et de l'image. C'est une formation aboutissant à un doublediplôme intitulé master "1+1 » entre une université chinoise, la South-East University (SEU) à Nankin (Chine) [1] et l'Université de Rennes 1, à Rennes (France) [2].
Dans la mesure où les établissements français se sont engagés depuis 2002 dans l'adaptation des licences et masters au processus de Bologne [3], ce diplôme est bâti sur les mêmes règles et plus particulièrement en termes de crédits (ECTS : European Credit for Transfer System). Les masters doivent correspondre à 120 crédits répartis normalement sur deux années, le cursus étant organisé en quatre semestres de 30 crédits chacun.

Le principal avantage de cette approche est de donner l’opportunité aux meilleurs étudiants étrangers qui sont sélectionnés dans ce cas, d'obtenir en complément de leur propre master un diplôme de master qui est reconnu dans toute l'Europe en raison de l'harmonisation effective du processus de Bologne depuis plusieurs années. Des efforts d'harmonisation ont d'ailleurs étaient menés au niveau des disciplines de l'EEA dans le cadre de programmes européens successifs, INEITMUCON [4], THEIERE [5], et EIE-SURVEYOR [6], menés au sein de l'association européenne, EAEEIE [7].

Dans la mesure où le programme proposé peut nécessiter des compétences qui ne sont pas forcément disponibles au plus haut niveau dans chacune des institutions, il peut être fait appel à des compétences d'institutions partenaires. C'est en particulier le cas dans le domaine de la fiabilité des composants et dispositifs microélectroniques, pour lequel des collègues de l'IMS de Bordeaux [8], les professeurs Yves Danto et Hélène Frémont, reconnus spécialistes internatio- 
naux du domaine, interviennent depuis de nombreuses années au master de Rennes.

La mise en place d'un tel projet n'en est pas moins délicate, puisqu'il faut adapter deux systèmes qui ne fonctionnent pas sur les mêmes règles de durée et de crédits des diplômes. Toutefois, la forte volonté réciproque a permis de mettre en place cette formation.

\section{MISE EN PLACE DU PROJET}

\subsection{Stratégie et opportunité}

Grâce à la volonté affichée des deux Présidents des universités concernées, et grâce à l'expérience déjà acquise dans des relations de recherche communes (Laboratoire international associé de recherche en imagerie médicale franco-chinois [9] entre la «SouthEast University», l'Université de Rennes 1, et l'organisme de recherche INSERM (Institut National de la Santé et de la Recherche Médicale) [10], les contacts ont été fortement facilités entre les équipes pédagogiques des deux établissements. Après l'organisation de seulement deux réunions plénières de part et d'autre avec les équipes pédagogiques, il a été possible de bâtir un projet réaliste en six mois environ.
Toutefois, la validation d'un diplôme étant une opération sérieuse, les démarches administratives qui ont suivies ont été plus longues puisqu’il est nécessaire, déjà du côté français de faire valider la proposition par la composante porteuse (UFR dans ce cas) puis par le Conseil des Études et de la Vie Universitaire, puis par le Conseil Scientifique et enfin par le Conseil d'Administration après avis de la Commission des Affaires Internationales et la validation par le service des affaires statutaires. Du côté chinois, la démarche peut apparaître plus simple, mais les différents services équivalents et les départements concernés sont également consultés.

Au moment de la construction du projet, les deux établissements avaient des masters qui fonctionnaient. A Rennes, un master en Électronique et Télécommunications comportant trois spécialités. La figure 1 montre la structure du master Électronique et Télécommunications de l'Université de Rennes 1 qui comporte trois spécialités : Conception et Technologie des Systèmes, (CTS), Systèmes pour Télécommunications (ST), et Signal-Image-Systèmes électroniques-Automatique (SISEA).

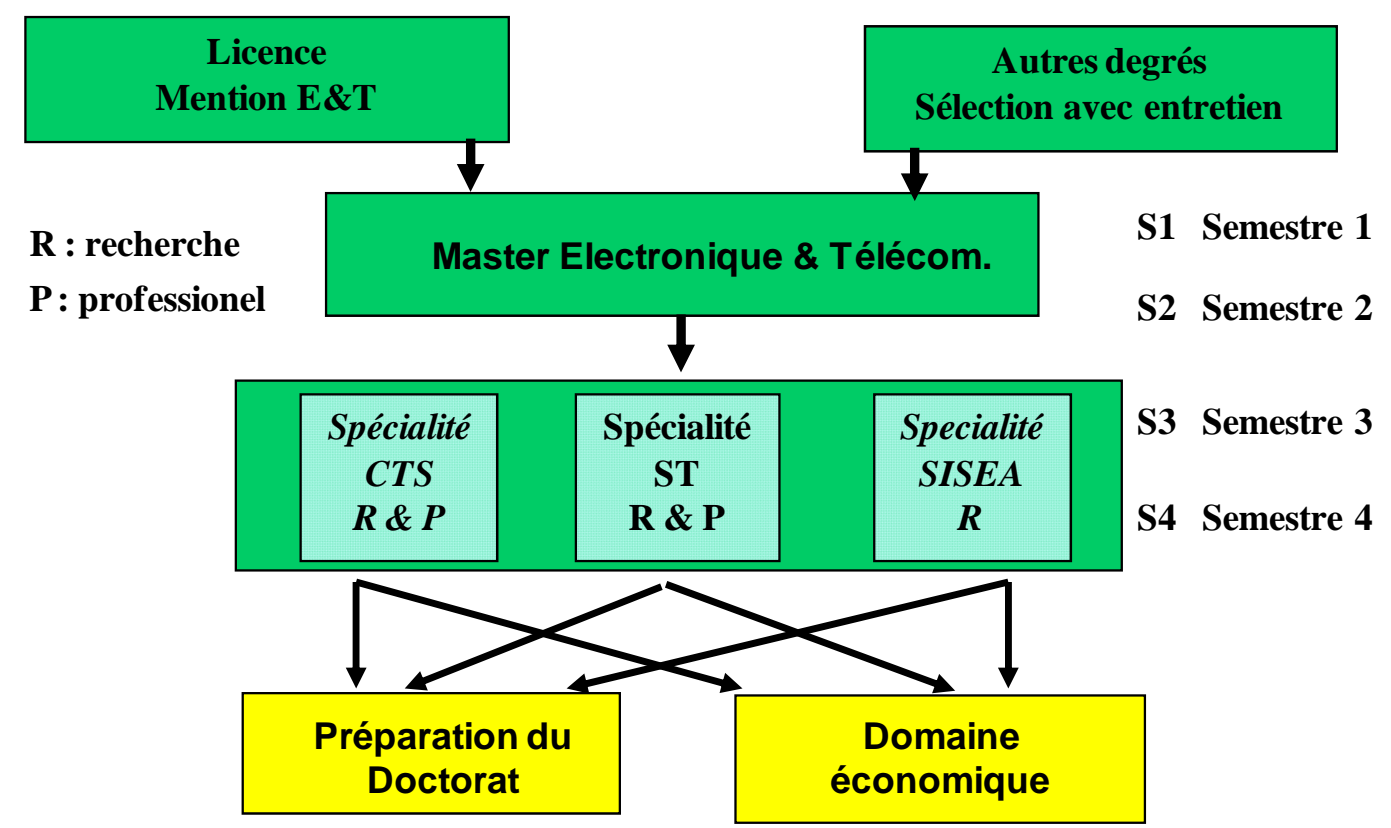

fig 1 : Structure du master Électronique et télécommunication de l'Université de Rennes 1. Trois spécialités sont ouvertes couvrant la microélectronique (CTS), les télécommunications (ST) le traitement du signal et de l'image (SISEA). CTS et SISEA ont été sélectionnées pour le projet de master international.

En pratique, deux spécialités ont été proposées pour l'extension à l'international : CTS dans le domaine de la microélectronique, et SISEA, dans le domaine du Signal et Traitement d'Images.

Le master chinois préparé par l'Université du Sud-Est à Nankin comporte quant à lui, huit spécialités. La structure est représentée figure 2. Ce master fonctionne normalement sur 5 semestres. Trois spécialités ont été sélectionnées pour le master commun ; ingénierie électronique, informatique et ingénierie biomédicale.

La présence de ces deux masters a constitué une forte opportunité pour la construction du projet commun grâce à l'existence de nombreux enseignements proches voire identiques des deux côtés et dans chacune des spécialités sélectionnés. 


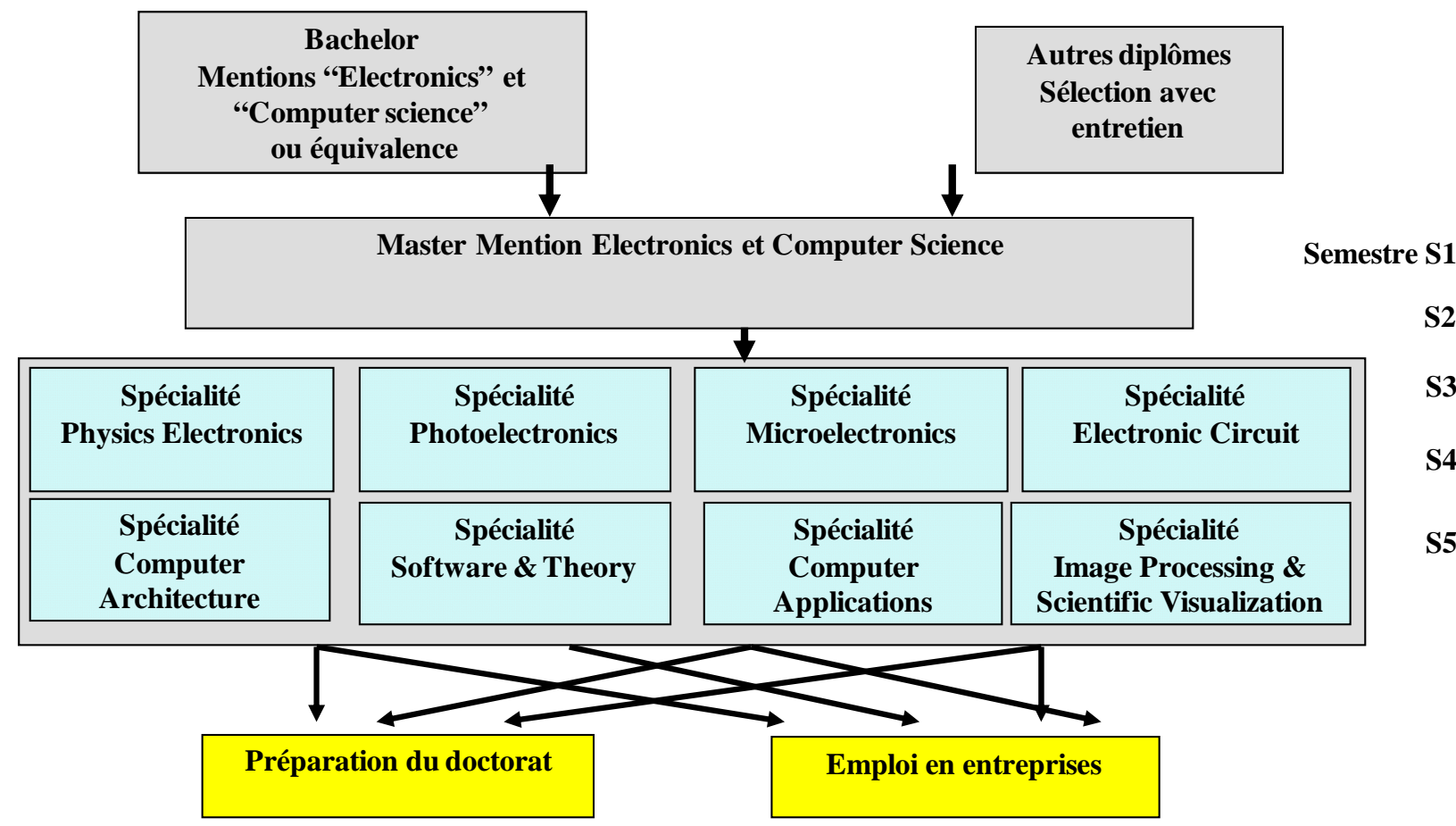

fig 2 : Structure du master à l' Université du Sud-Est à Nankin. Huit spécialités sont ouvertes. Trois ont été sélectionnées pour le projet de master international.

\subsection{Adaptation des durées et programmes}

Pour contourner les problèmes linguistiques, tous les enseignements sont assurés en anglais. Les étudiants sont sélectionnés sur la base de leur connaissance scientifique et de leurs résultats au « Bachelor » chinois, mais aussi de leur maîtrise théorique et pratique de la langue anglaise.

Le programme pédagogique a été construit sur la base des compétences réciproques des équipes pédagogiques afin de bénéficier au maximum des meilleures interventions dans un domaine spécialisé donné. Ainsi, les enseignements dispensés et les crédits sont équilibrés entre les deux partenaires.

Étant donné que le diplôme de Bachelor est d'une durée de quatre années en Chine, certains étudiants ont déjà reçu dans la spécialité concernée, certains enseignements correspondant aux programmes de la première année des masters français. Ainsi, il a été convenu de valider certains modules, correspondant à des crédits ECTS et permettant de focaliser le programme pédagogique sur les spécialités importantes du master. La figure 3 donne un aperçu de l'approche, les crédits intitulés M1-A étant considérés comme acquis, les crédits suivants étant assurés par l'équipe pédagogique mixte.

Pour tout étudiant du master $1+1$, les enseignements du master chinois sont obligatoires et un effort supplémentaire est demandé pour l'acquisition du diplôme européen. La figure 3 compare les deux systèmes et décrit les bases ayant permis le montage du projet. L’objectif est d'aboutir aux 120 crédits.
Adaptation du master entre SEU et UR1

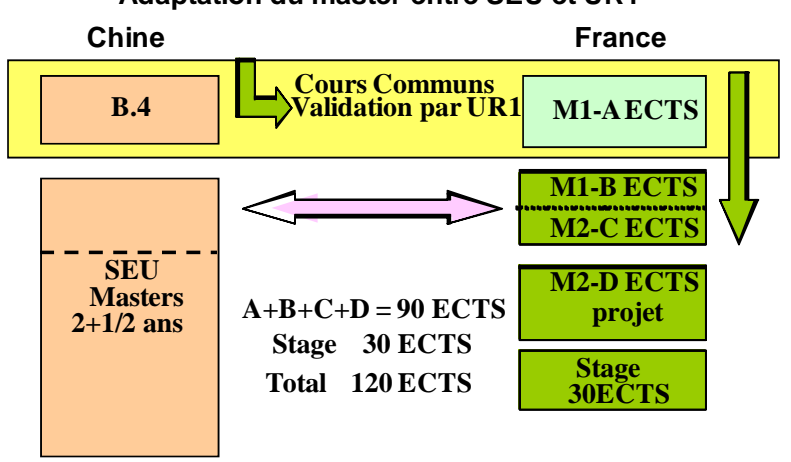

fig 3 : Comparaison de deux systèmes de formation, Chinois et français. L'université du Sud-Est (SEU) prépare à un bachelor en 4 ans puis un master en 2 ans et demi. Le processus de Bologne prévoit une structure $3+2$ (licence puis master).

Un autre problème important à résoudre porte sur la durée normale du diplôme qui est officiellement de deux ans et demi en Chine. Pour le master, chinois, la durée normale du stage de fin d'étude est d'un an et demi, soit une année de plus que pour les étudiants français. Les enseignements complémentaires assurés par le partenaire français de seconde année de master se déroulent ainsi sur des courtes durées (en général d'une semaine) prélevées sur la seconde année du master chinois, c'est-à-dire durant leur stage de longue durée. Cette option facilite l'organisation logistique puisque les obligations des étudiants sont moindres en raison de l'absence d'autres enseignements obligatoires. 
La seule contrainte supplémentaire vient donc de la durée du double diplôme. Afin de répondre aux exigences du diplôme chinois, la durée effective du stage de master 2 est allongée d'un semestre. En pratique, la délivrance du diplôme côté français est conditionnée par l'obtention simultanée du diplôme côté chinois.

\subsection{Jury et attribution du diplôme}

Le jury doit être en adéquation avec les deux structures partenaires. Il est ainsi construit avec une parité des deux institutions. Il est constitué de six enseignants impliqués dans les enseignements communs aux deux institutions. Pour l’Université de Rennes 1, le Président du jury doit être un professeur en poste dans cet établissement. La même règle est appliquée pour le partenaire chinois.

\subsection{Plaquette de présentation du diplôme}

La maquette du diplôme a été construite sur la base des spécialités proposées. La figure 2 montre la plaquette qui a été conçue pour mettre en valeur les deux spécialités du diplôme et donc pour promouvoir auprès des meilleurs étudiants de Nankin ce double diplôme.

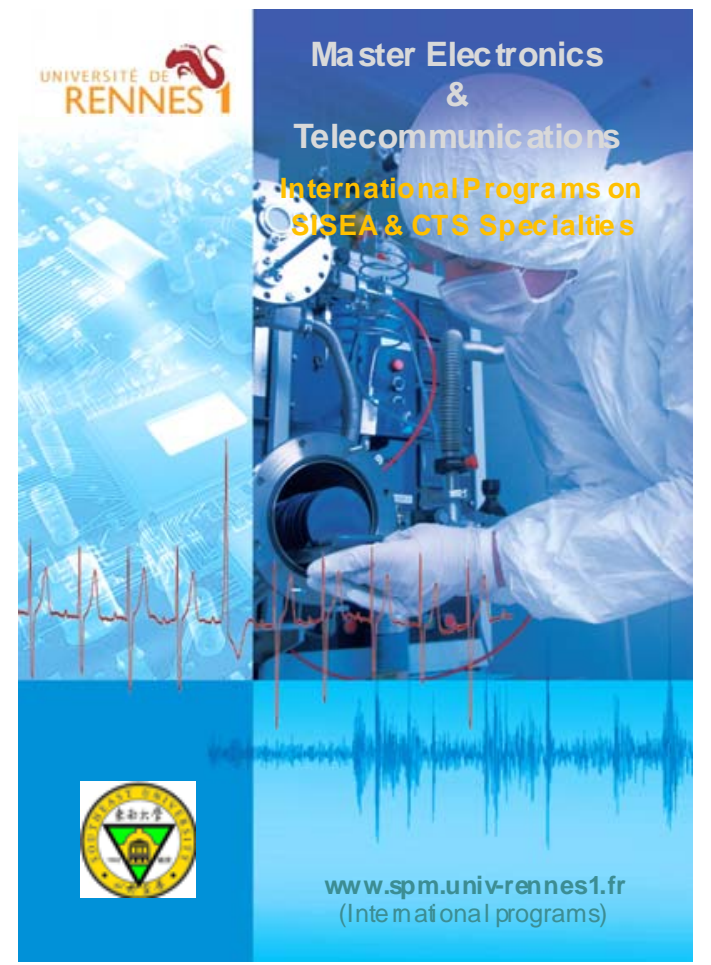

fig 4 : Plaquette de présentation du programme de master international entre les deux universités, SEU and UR1, pour les deux spécialités. Cette plaquette comporte sur les 3 autres pages la présentation du cursus et les principaux intervenants.

\section{DÉFINITION DU CONTENU}

Le contenu des cours a été construit sur la base des deux programmes initiaux et comme déjà annoncé dans les deux spécialités suivantes : microélectronique
(CTS) et signal et traitement des images (SISEA). Du côté de Nankin, trois départements sont impliqués ; l'Informatique, l’Ingénierie Biomédicale et Électronique et Microsystèmes. Les prérequis ont été vérifiés par les deux partenaires. Certains cours sont ainsi communs à la première année, et constituent le tronc commun du diplôme pour les deux spécialités. La seconde année est entièrement dédiée à la spécialité, soit microélectronique, soit traitement du signal et images. Les cours proposés sont ainsi répartis ; leurs titres sont donnés en anglais, ce qui correspond à la réalité du programme.

1) Microelectronics: Elements of Information Technologies - Development of Electronic Science Embedded Systems - RF \& HF Electronics - Power Electronics - Microtechnologies and Sensors - System Design - Mixt and HF circuit Design - Advanced Embedded systems - Technologies of Devices - Test and Instrumentation - Microtechnological sensors and Biosensors - MEMs - Quality and Reliability

2) Signal and Image Processing : Algorithm design - Process control and vision - Advanced Signal Processing - Computer Vision - Software Design - Detection and Estimation - Kalman Markov Filtering - Optimization - Classification and Fusion - Adaptive Filtering and Filter banks - Computer Science - Image Processing and Wavelets - Geometrical Modeling and Statistics - Biomedical Engineering and Data mining Pattern Recognition and Image Fusion - Image Sequence Analysis

Pour ces deux spécialités, chaque institution dispense approximativement la moitié des cours. Bien évidemment, la responsabilité des modules incombe aux collègues reconnus compétents dans le domaine concerné afin d'aboutir au meilleur niveau scientifique possible.

\section{ASPECT FINANCIER DE LA FORMATION}

Sur le plan financier, les deux établissements contribuent aux dépenses effectives en termes de coût de personnels et de dépenses de fonctionnement.

Toutefois, l'Université du Sud-Est de Nankin fait un effort supplémentaire en contribuant aux dépenses des missions des enseignants envoyés par l’Université de Rennes 1, incluant voyage et logement sur place.

Afin de limiter ces dépenses, sur le plan pratique, les enseignants viennent sur une durée minimale d'une semaine et dispensent autour de 20 heures de cours. Cela nécessite une organisation précise et suffisamment prédictive aussi bien au niveau de l'emploi du temps des étudiants que pour les moyens de transport. Ce choix minimise les coûts de transport et mission mais affecte en partie l'approche pédagogique ; en effet, un très grand flux d'information dans une courte durée est un exercice difficile aussi bien pour les étudiants que pour les enseignants. Toutefois, cette approche constitue aussi un apprentissage à des situations rencontrées dans la vie professionnelle lors de formations complémentaires spécialisées. Cette expérience est donc enrichissante pour les étudiants. Cette ap- 
proche permet par ailleurs une ouverture à des formations ciblées, notamment dans le cadre de la formation continue.

\section{BILAN APRES 2 ANNÉES D'EXPERIENCE}

\subsection{Bilan quantitatif et attractivité}

Ce master a été ouvert au cours de l'année académique 2008-2009. Après plus de deux années de fonctionnement, les résultats sont très intéressants tant sur le plan des étudiants formés que sur l'aspect pédagogique.

La première promotion a comporté 17 étudiants, 11 en spécialité Signal et Image et 6 en Microélectronique. La deuxième promotion a attiré 34 étudiants répartis respectivement en 19 et 15 . La troisième a attiré 29 étudiants tous sélectionnés parmi les meilleurs étudiants des bachelors suite au concours national d'entrée en master. L'attractivité de ce double diplôme est donc devenue importante.

Afin d'accroître cette attractivité, la direction de l’Université du Sud-Est a souhaité que certains bons étudiants puissent effectuer au cours de leur master un séjour en France afin d'une part de connaître un tant soit peu la culture française mais aussi de bénéficier de l'accès aux plateformes technologiques accessibles aux étudiants français, en particulier dans le domaine de la microélectronique. En effet, le pôle rennais du CNFM [11], le CCMO [12] dispose d'une salle blanche dans laquelle des réalisations technologiques sont organisées pour de nombreuses formations françaises. De plus, l'IETR [13], institut de recherche dispose d'une salle anéchoïde permettant de faire avec des étudiants des caractérisations et simulation dans le domaine des hautes fréquences. Les étudiants de la spécialité Microélectronique ont pu bénéficier d'un stage de près de 4 semaines en France qui a été très fortement apprécié par tous les étudiants concernés.

Il peut être noté que la richesse de cette expérience a conduit la direction de l'Université du Sud-Est à souhaiter le renouvellement de cette expérience en 2011 pour la seconde promotion. Cette université prendra en charge les missions des étudiants et souhaite de plus utiliser une seconde plateforme du pôle CNFM à Bordeaux [14] durant le même séjour.

\subsection{Résultats finaux de la première promotion}

Le premier jury de fin de master s'est tenu en novembre 2010. A l'exception d'un étudiant qui a changé de sujet de stage en cours de deuxième année et qui n'avait pas encore validé ce nouveau stage, tous les étudiants ont été reçu en première session avec mention bien ou très bien. La première promotion a été exceptionnelle en qualité et homogénéité.

Quant aux étudiants en fin de première année de master, ils n'ont eu aucun problème pour valider leurs 60 crédits de première année en 2010. Il est clair que la très dure sélection à l'entrée garantit l'excellence des étudiants et leur motivation.

\subsection{Retour d'expérience sur le plan pédagogique}

Une enquête en fin de cursus auprès des diplômés a montré plusieurs aspects intéressants.

Le premier point concerne l'adaptation à la pédagogie. Tous les enseignants devaient dispenser leurs cours en anglais qui n'était pas leur langue maternelle. Cela sous-entend une attention particulière sur l'utilisation d'expressions orales claires, dans un flot relativement lent, avec une construction logique. Il faut aussi des supports visuels bien documentés et suffisamment riches en vocabulaire qui peut être défini ou précisé. Il faut bien utiliser les symboles et notations qui doivent être éventuellement redéfinis en relation avec les études antérieures. L'encouragement des étudiants à établir le contact et le dialogue afin de révéler les points délicats fait aussi partie de la démarche.

Le second point est relatif à la coordination et l'adaptation des deux institutions afin de prendre en compte les différences relatives aux méthodes pédagogiques. Au niveau du master, les enseignants français des domaines concernés cherchent à donner une vue d'ensemble scientifique des problèmes plutôt que des démonstrations et des calculs que les étudiants sont censés savoir mener. Il s'agit aussi de susciter un travail plus orienté vers la compréhension que vers l'apprentissage par cœur de formules et recettes. Lors des premières leçons, cette approche a été déroutante pour certains étudiants plus habitués au bachotage et à la mémorisation dans la préparation de leur diplôme d'entrée en master. Certains enseignements font appel à une de solides connaissances transversales couvrant de nombreux domaines qui ont été jusqu’alors étudiés séparément. Pour exemple, la technologie microélectronique fait appel à des connaissances en chimie, mathématiques, physique quantique, optique, mécanique, statistique, traitement du signal, physique du solide, physique des composants, électronique. Les enseignants sont ainsi amenés à construire une nouvelle approche qui implique des méthodes pédagogiques adaptées à l'auditoire. Si cela semble naturel pour un enseignant, l'exercice en langue étrangère n'en reste pas moins une difficulté à surmonter. Les premières heures de cours constituent en fait un observatoire réciproque et une expérience pédagogique enrichissante.

Toutefois à ce niveau d'étude et en raison de la très forte motivation des étudiants et de leurs capacités potentielles très élevées, les résultats deviennent très rapidement positifs et créent un climat réciproque de confiance entre étudiants et professeurs.

\section{CONCLUSION}

Cette expérience s'est avérée concluante et pourrait servir de référence dans la démarche de création de formations similaires. Les deux partenaires institutionnels travaillent actuellement pour développer des formations dans d'autres disciplines. Il faut cependant savoir que le plus important reste la volonté et la compréhension réciproques des partenaires universitaires 
qui doivent y voir, à juste titre, un énorme enrichissement collectif et partagé.

\section{Remerciements}

Les auteurs souhaitent remercier les Présidents et les services administratifs des études et des relations internationales des deux universités partenaires ainsi que les équipes pédagogiques des deux spécialités pour la mise en place et le fonctionnement de ce master.

\section{Bibliographie}

[1] see http://www.seu.edu.cn.

[2] see http://www.univ-rennes1.fr

[3] «The Bologna Declaration on the European space for higher education: an explanation, 1999 [online]: http://europa.eu.int/comm/education/policies/educ/bolog na/bologna.pdf

[4] Project INEIT-MUCON : Innovations for Education in Information Technology through Multimedia and Communication Networks www.eaeeie.org/ineitmucon/index02.html
[5] Project THEIERE. Thematic Harmonisation in Electrical and Information EngineeRing in Europe www.eaeeie.org/theiere/THEIERE_monograph.../THEIE RE.pdf

[6] Thiriet, J.M. Robert, M. Martins, M.J. Ward, A.E. Bonnaud, O. Hoffmann, M. Yahoui, H. Fremont, H. « New results for the EIE-Surveyor project » EAEEIE Annual Conference, 2008, Print ISBN: 978-1-4244-2008-7, pp. $104-107$

[7] EAEEIE : European Association for Education i Electrical and Information Engineering, http://www.eaeeie.org/

[8] http://www.ims-bordeaux..fr/

[9] Centre de recherche en information biomédicale sinofrançais (CRIBs) http://www.univrennes1.fr/themes/actualites/uneActualite/?contentId=574 01

[10] http://www.inserm.fr/

[11] CNFM : Coordination National en Microélectronique et Nanotechnologies : www.cnfm.fr

[12] CCMO : Centre Commun de Microélectronique de l'Ouest : ccmo.univ-rennes1.fr

[13] IETR : Institut d'Électronique et Télécommunications de Rennes, UMR CNRS 6164 : www.ietr.univ-rennes1.fr

[13] PCB : Pôle CNFM de Bordeaux 